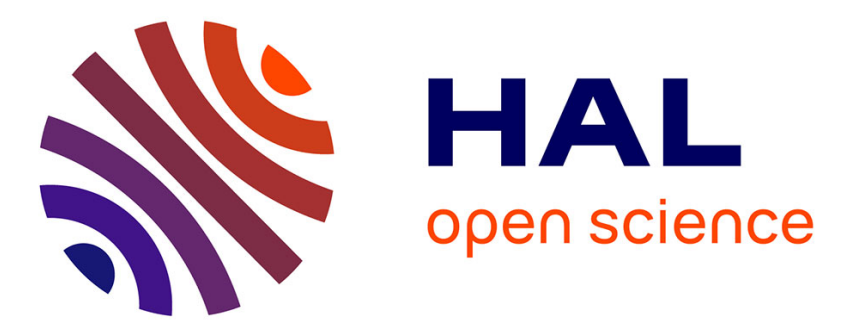

\title{
Credit segmentation in general equilibrium
}

Sebastián Cea-Echenique, Juan Pablo Torres-Martínez

\section{To cite this version:}

Sebastián Cea-Echenique, Juan Pablo Torres-Martínez. Credit segmentation in general equilibrium. 2015. hal-01151576v2

\section{HAL Id: hal-01151576 \\ https://hal.science/hal-01151576v2}

Submitted on 11 Dec 2015

HAL is a multi-disciplinary open access archive for the deposit and dissemination of scientific research documents, whether they are published or not. The documents may come from teaching and research institutions in France or abroad, or from public or private research centers.
L'archive ouverte pluridisciplinaire HAL, est destinée au dépôt et à la diffusion de documents scientifiques de niveau recherche, publiés ou non, émanant des établissements d'enseignement et de recherche français ou étrangers, des laboratoires publics ou privés. 


\section{Documents de Travail du
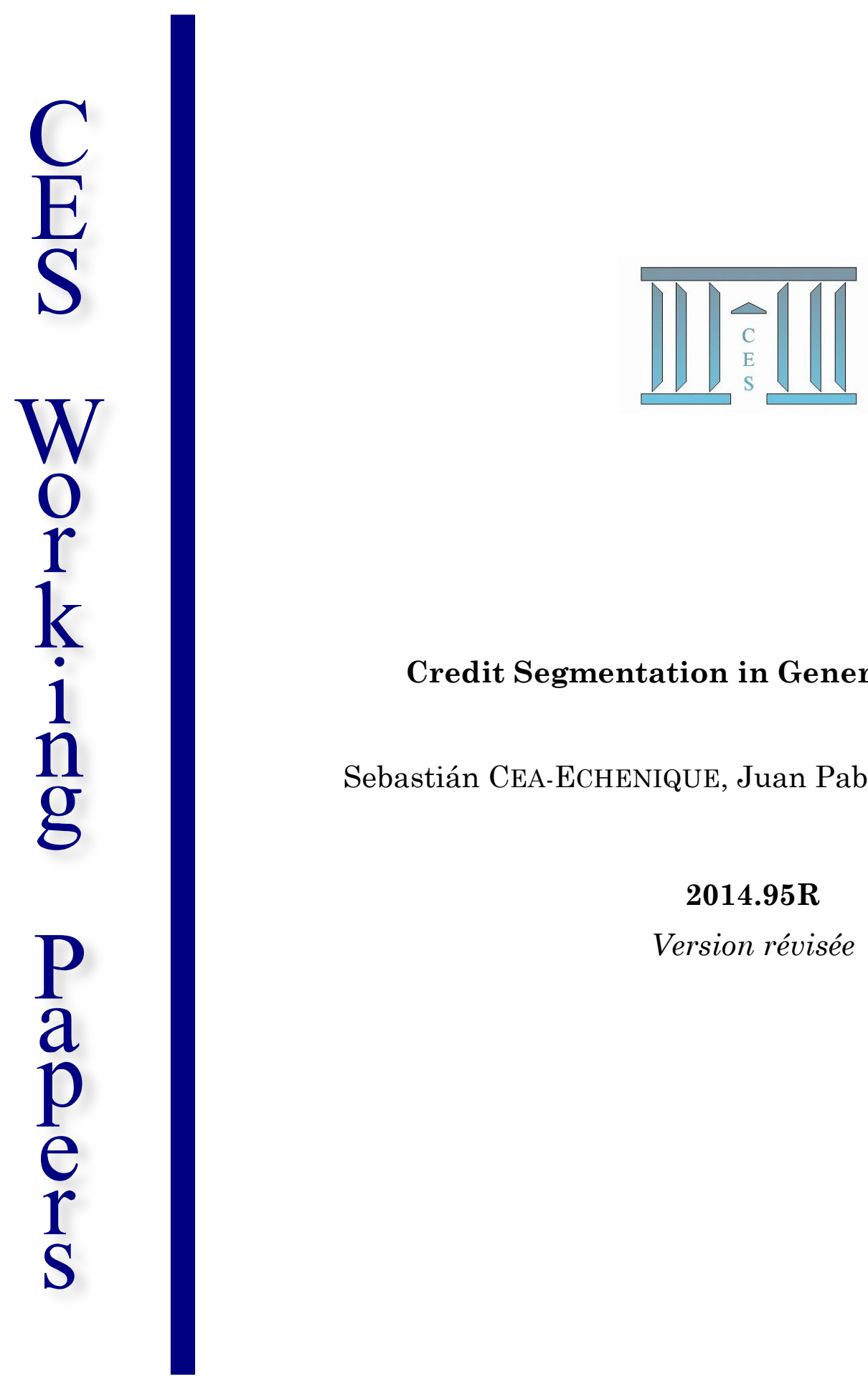

Credit Segmentation in General Equilibrium

Sebastián CEA-Echenique, Juan Pablo ToRRes-MARTínez

2014.95R

Version révisée

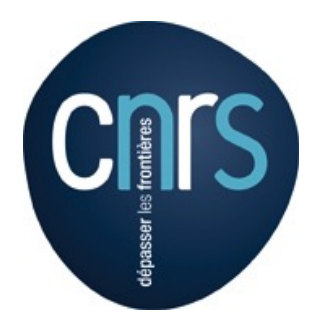




\title{
CREDIT SEGMENTATION IN GENERAL EQUILIBRIUM
}

\author{
SEBASTIÁN CEA-ECHENIQUE AND JUAN PABLO TORRES-MARTÍNEZ
}

\begin{abstract}
We build a general equilibrium model with endogenous borrowing constraints compatible with credit segmentation. There are personalized trading restrictions connecting prices with both portfolio constraints and consumption possibilities, a setting which has not thoroughly been addressed by the literature. Our approach is general enough to be compatible with incomplete market economies where there exist wealth-dependent and/or investment-dependent credit access, borrowing constraints precluding bankruptcy, or assets backed by physical collateral.

To prove equilibrium existence, we assume that both investment on segmented assets is not required to obtain access to credit and transfers implementable in segmented markets can be superreplicated by investing in non-segmented markets. For instance, this super-replication property is satisfied if either (i) all individuals have access to borrow at a risk-free rate; or (ii) financial contracts make real promises in terms of non-perishable commodities; or (iii) promises are backed by physical collateral.
\end{abstract}

KEywords. Incomplete Markets; General Equilibrium; Endogenous Trading Constraints.

JEL Classification. D52, D54.

\section{INTRODUCTION}

The differentiated access to commodity or asset markets endogenously emerges due to regulatory or institutional considerations. As a consequence, several kinds of trading restrictions are observed in financial markets: margin calls, collateral requirements, consumption quotas or income-based access to funding, among others. With the aim of understanding the effects of those restrictions in competitive markets, a vast literature of general equilibrium has been developed. That research

Date: July 25, 2015.

We are grateful to Antonela Racca for several suggestions and comments. The authors acknowledge financial support from Conicyt-Chile through Fondecyt project 1120294. Cea-Echenique is grateful to Conicyt-Chile from financial support. This research was partly undertaken while the authors visiting the University of Salamanca.

Sebastián Cea-Echenique

Paris School of Economics - University Paris 1

e-mail: sebastian.cea@psemail.eu

Juan Pablo Torres-Martínez

Department of Economics, Faculty of Economics and Business, University of Chile e-mail: juan.torres@fen.uchile.cl. 
has given consideration to models where financial trade is restricted by fixed, price-dependent, or consumption-dependent portfolio constraints. Nevertheless, channels connecting prices with both portfolio constraints and consumption possibilities have not thoroughly been addressed by the literature. The objective of this paper is to contribute to this direction.

We analyze the existence of equilibria in a two period economy where agents are subject to price-dependent credit constraints that affect the access to physical and financial markets. Our approach is general enough to be compatible with incomplete market economies where there exists, for instance, wealth-dependent and/or investment-dependent credit access, borrowing constraints precluding bankruptcy, and assets backed by physical collateral.

In order to make credit segmentation compatible with the existence of a competitive equilibrium, we assume that both investment on segmented assets is not required to obtain access to credit and assets payments can be super-replicated either by deliveries of non-perishable commodities or by the promises of assets that all agents can short-sale. For instance, this latter property holds when either (i) individuals have access to borrow resources through a portfolio making positive payments at all states of nature where remaining assets pay (e.g. a risk-free asset); or (ii) all assets are real and promises are measured in units of non-perishable commodities; or (iii) debts are backed by physical collateral. In addition to our results of equilibrium existence, we provide examples clarifying the relevance of the super-replication assumption.

The related literature is described in the next section. In Sections 3 and 4 we state our model and introduce the basic assumptions over individuals' characteristics and trading constraints. Sections 5 is devoted to discuss our main assumptions and results, whose proofs are given in the Appendix.

\section{INSERTION IN THE LITERATURE}

The existence of competitive equilibria was deeply studied in incomplete markets models where agents are subject to exogenous portfolio constraints. The case of portfolio restrictions determined by linear equality constraints is addressed by Balasko, Cass and Siconolfi (1990) for nominal assets, and by Polemarchakis and Siconolfi (1997) for real assets. When portfolio restrictions are determined by convex and closed sets containing zero, the case of nominal or numéraire assets is studied by Cass (1984, 2006), Siconolfi (1989), Cass, Siconolfi and Villanacci (2001), Martins-daRocha and Triki (2005), Won and Hahn (2007, 2012), Aouani and Cornet (2009, 2011), and Cornet and Gopalan (2010). In the same context, the case of real assets is analyzed by Radner (1972), Angeloni and Cornet (2006), and Aouani and Cornet (2011). In general terms, these authors prove equilibrium existence requiring non-redundancy hypotheses over financial structures and/or financial survival requirements. Under these assumptions, individuals' allocations and asset prices can be endogenously bounded without inducing frictions in the model. 
There are several results that include price-dependent portfolio constraints in nominal or real assets markets. These models assume that financial constraints are determined by a finite number of inequalities, and use differentiable techniques to ensure the existence of equilibrium and to analyze its stability and local-uniqueness. In this context, equilibrium existence is addressed by Carosi, Gori and Villanacci (2009) for numéraire asset markets with portfolio constraints, by Gori, Pireddu and Villanacci (2013) for numéraire and real asset markets with borrowing constraints, and by Hoelle, Pireddu, and Villanacci (2012) for real asset markets with wealth-dependent credit limits.

In addition to these approaches, Seghir and Torres-Martínez (2011) propose a model where trading constraints restrict the access to debt in terms of first-period consumption. Financial survival conditions are not required. However, they assume that preferences are such that individuals may compensate the losses on well-being generated by reductions of future consumption with increments in present demand.

We contribute to this literature with a model where borrowing constraints make the access to liquidity dependent on prices, investment, and consumption. Since we want to make trading constraints compatible with credit market segmentation, we do not impose financial survival conditions. Also, financial constraints are not necessarily determined by a finite number of inequalities and individual's preferences are not restricted by differentiable assumptions or by impatience conditions as in Seghir and Torres-Martínez (2011). Alternatively, we suppose that assets payments can be super-replicated either by deliveries of non-perishable commodities or by the promises of assets that all agents can short-sale.

\section{MODEL}

We consider a two-period economy with uncertainty about the realization of a state of nature in the second period, which belongs to a finite set $S$. Let $\mathcal{S}=\{0\} \cup S$ be the set of states of nature in the economy, where $s=0$ denotes the unique state at the first period.

There is a finite set $\mathcal{L}$ of perfectly divisible commodities, which are subject to transformation between periods and can be traded in spot markets at prices $p=\left(p_{s}\right)_{s \in \mathcal{S}} \in \mathbb{R}_{+}^{\mathcal{L} \times \mathcal{S}}$. We model the transformation of commodities between periods by linear technologies $\left(Y_{s}\right)_{s \in S}$. Thus, a bundle $y \in \mathbb{R}_{+}^{\mathcal{L}}$ demanded at the first period is transformed, after its consumption and the realization of a state of nature $s \in S$, into the bundle $Y_{s} y \in \mathbb{R}_{+}^{\mathcal{L}}$.

There is a finite set $\mathcal{J}$ of financial contracts available for trade at the first period that make promises contingent to the realization of uncertainty. Let $q=\left(q_{j}\right)_{j \in \mathcal{J}} \in \mathbb{R}_{+}^{\mathcal{J}}$ be the vector of asset prices and denote by $R_{j}(p)=\left(R_{s, j}(p)\right)_{s \in S} \in \mathbb{R}_{+}^{S}$ the vector of payments associated to asset $j \in \mathcal{J}{ }^{1}$

\footnotetext{
${ }^{1}$ Our financial structure is general enough to be compatible with several types of assets. For instance, to include a nominal asset $j$ it is sufficient to assume that there is $\left(N_{s, j}\right)_{s \in S} \in \mathbb{R}_{+}^{S}$ such that $R_{s, j} \equiv N_{s, j}, \forall s \in S$. To include a real asset $k$ we can define payments $R_{s, k}(p)=p_{s} \cdot A_{s, k}, \forall s \in S$, where $\left(A_{s, k}\right)_{s \in S} \in \mathbb{R}_{+}^{\mathcal{L} \times S}$.
} 
For notation convenience, let $\mathbb{P}:=\left(\mathbb{R}_{+}^{\mathcal{L}} \times \mathbb{R}_{+}^{\mathcal{J}} \backslash\{0\}\right) \times\left(\mathbb{R}_{+}^{\mathcal{L}} \backslash\{0\}\right)^{S}$ be the space of commodity and asset prices $\left(\left(p_{0}, q\right),\left(p_{s}\right)_{s \in S}\right)$ satisfying $\left(p_{0}, q\right) \neq 0$ and $p_{s} \neq 0, \forall s \in S$. Also, let $\mathbb{E}:=\mathbb{R}_{+}^{\mathcal{L} \times \mathcal{S}} \times \mathbb{R}^{\mathcal{J}}$ be the space of consumption and portfolio allocations.

There is a finite set $\mathcal{I}$ of consumers that may trade assets in order to smooth their consumption. Each agent $i \in \mathcal{I}$ has a utility function $V^{i}: \mathbb{R}_{+}^{\mathcal{L} \times \mathcal{S}} \rightarrow \mathbb{R}$ and endowments $w^{i}=\left(w_{s}^{i}\right)_{s \in \mathcal{S}} \in \mathbb{R}_{+}^{\mathcal{L} \times \mathcal{S}}$. Let $W^{i}=\left(W_{s}^{i}\right)_{s \in \mathcal{S}}:=\left(w_{0}^{i},\left(w_{s}^{i}+Y_{s} w_{0}^{i}\right)_{s \in S}\right)$ be the aggregated endowments of agent $i \in \mathcal{I}$.

Each individual $i$ is subject to personalized trading constraints, which are determined by a correspondence $\Phi^{i}: \mathbb{P} \rightarrow \mathbb{E}$. Notice that, agents may be subject to endogenous borrowing constraints, as the access to liquidity can depend on prices, investment and consumption. We assume that there are no restrictions on investment, i.e., $\Phi^{i}(p, q)+\mathbb{R}_{+}^{\mathcal{L} \times \mathcal{S}} \times \mathbb{R}_{+}^{\mathcal{J}} \subseteq \Phi^{i}(p, q), \forall(p, q) \in \mathbb{P}, \forall i \in \mathcal{I}^{2}$

Given prices $(p, q) \in \mathbb{P}$, each agent $i$ chooses a consumption bundle $x^{i}=\left(x_{s}^{i}\right)_{s \in \mathcal{S}}$ and a portfolio $z^{i}=\left(z_{j}^{i}\right)_{j \in \mathcal{J}}$ in her choice set $C^{i}(p, q)$, which is characterized by the vectors $\left(x^{i}, z^{i}\right) \in \Phi^{i}(p, q)$ satisfying the following budget restrictions:

$$
p_{0} \cdot x_{0}^{i}+q \cdot z^{i} \leq p_{0} \cdot w_{0}^{i} ; \quad p_{s} \cdot x_{s}^{i} \leq p_{s} \cdot\left(w_{s}^{i}+Y_{s} x_{0}^{i}\right)+\sum_{j \in \mathcal{J}} R_{s, j}(p) z_{j}^{i}, \quad \forall s \in S .
$$

Definition 1. A vector $\left((\bar{p}, \bar{q}),\left(\bar{x}^{i}, \bar{z}^{i}\right)_{i \in \mathcal{I}}\right) \in \mathbb{P} \times \mathbb{E}^{\mathcal{I}}$ is a competitive equilibrium for the economy with endogenous trading constraints when the following conditions hold:

(i) Each agent $i \in \mathcal{I}$ maximizes her preferences, $\left(\bar{x}^{i}, \bar{z}^{i}\right) \in \operatorname{argmax}_{\left(x^{i}, z^{i}\right) \in C^{i}(\bar{p}, \bar{q})} V^{i}\left(x^{i}\right)$.

(ii) Individuals' plans are market feasible, $\sum_{i \in \mathcal{I}}\left(\bar{x}^{i}-W^{i}, \bar{z}^{i}\right)=0$.

Our objective is to determine conditions that make price-dependent trading constraints $\left\{\Phi^{i}\right\}_{i \in \mathcal{I}}$ compatible with equilibrium existence, even in the presence of credit market segmentation, which means that there are contracts that not all agents can short-sale, i.e.,

$$
\left\{j \in \mathcal{J}: \exists i \in \mathcal{I}, \quad\left(x^{i}, z^{i}\right) \in \Phi^{i}(p, q) \Longrightarrow z_{j}^{i} \geq 0, \forall(p, q) \in \mathbb{P}\right\} \neq \emptyset .
$$

Notice that, the existence of credit market segmentation is not compatible with financial survival. ${ }^{3}$ This assumption requires, independent of prices, that all agents have access to liquidity at the first

\footnotetext{
${ }^{2}$ At the cost of additional complexity, our model could be generalized to include price-dependent investment constraints (see Cea-Echenique and Torres-Martínez (2014)).

${ }^{3}$ Some authors impose a financial survival condition which is independent of physical endowments, although is still incompatible with credit segmentation: given non-arbitrage financial prices, all agents have access to some amount of liquidity by short-selling promises (see, for instance, Aouani and Cornet (2009, Assumption FN2), Aouani and Cornet (2011, Assumption FS)).
} 
period by either selling physical endowments or short-selling assets:

$$
\bigcap_{(p, q) \in \mathbb{P}}\left\{i \in \mathcal{I}: \exists\left(x^{i}, z^{i}\right) \in \Phi^{i}(p, q), p_{0} \cdot w_{0}^{i}-q \cdot z^{i}>0\right\}=\mathcal{I} .^{4}
$$

Our approach to trading constraints is general enough to include, as particular cases, exogenous borrowing constraints or price-dependent borrowing constraints. More precisely, an agent $i$ is subject to exogenous borrowing constraints if $\Phi^{i}(p, q)=\mathbb{R}_{+}^{\mathcal{L} \times \mathcal{S}} \times Z^{i}$, where $Z^{i}+\mathbb{R}_{+}^{\mathcal{J}} \subseteq Z^{i}$. Alternatively, she is burden by price-dependent borrowing constraints when $\Phi^{i}(p, q)=\left\{\left(x^{i}, z^{i}\right) \in \mathbb{E}: z^{i}+g_{k}^{i}(p, q) \geq\right.$ $\left.0, \forall k \in\left\{1, \ldots, m_{i}\right\}\right\}$, where $g_{k}^{i}: \mathbb{P} \rightarrow \mathbb{R}_{+}^{\mathcal{J}}$, for every $i \in \mathcal{I}$ and $k \in\left\{1, \ldots, m_{i}\right\}$.

Example 1. Suppose that $\mathcal{J}=\left\{j_{1}, j_{2}, j_{3}\right\}$ and, for each $(p, q) \in \mathbb{P}$ and $i \in \mathcal{I}$, we have that

$$
\left(x^{i}, z^{i}\right) \in \Phi^{i}(p, q) \Longleftrightarrow\left\{\begin{aligned}
z_{j_{1}}^{i} & \in\left[\min \left\{p_{0} \cdot\left(\tau_{1}-w_{0}^{i}\right), 0\right\},+\infty\right) \\
z_{j_{2}}^{i} & \in\left[\min \left\{p_{0} \cdot\left(w_{0}^{i}-\tau_{2}\right), 0\right\},+\infty\right) \\
z_{j_{3}}^{i} & \in\left[\min \left\{K-\sum_{k \in \mathcal{J}^{\prime}} q_{k} z_{k}^{i}, 0\right\},+\infty\right)
\end{aligned}\right.
$$

where $\tau_{1}, \tau_{2} \in \mathbb{R}_{+}^{\mathcal{L}}, K>0$ and $\mathcal{J}^{\prime} \subseteq \mathcal{J} \backslash\left\{j_{3}\right\}$.

Then, it follows that $j_{1}$ is a credit line available for high income agents, because agent $i$ can short-sale it if and only if the value of her first period endowment is greater than the threshold $p_{0} \tau_{1}$. Analogously, only low-income agents can short-sale asset $j_{2}$. In addition, the access to credit through asset $j_{3}$ depends on the amount of investment in some financial contracts, i.e., only investors expending an amount greater than $K$ in assets belonging to $\mathcal{J}^{\prime}$ have access to short-sale $j_{3}$.

EXAMPLE 2. Since trading constraints may induce restrictions on consumption, we can allow for derivative contracts as commodity options. Indeed, let $j \in \mathcal{J}$ be a financial contract such that, for every $(p, q) \in \mathbb{P}$ and $i \in \mathcal{I}$,

$$
R_{s, j}(p)=\max \left\{Y_{s} y-K, 0\right\}, \forall s \in S ; \quad\left(x^{i}, z^{i}\right) \in \Phi^{i}(p, q) \Longrightarrow x_{0}^{i}+\kappa y \min \left\{z_{j}^{i}, 0\right\} \geq 0
$$

where $y \in \mathbb{R}_{+}^{\mathcal{L}}, K>0$ and $\kappa \in[0,1)$. Then, $j$ is a commodity option that gives the right to buy in the second period, at a strike price $K$, the bundle obtained by the transformation of $y$ through time. To short-sell this option, agents are required to buy a portion $\kappa$ of $y$ as guarantee.

\footnotetext{
${ }^{4}$ Since financial survival is a property defined on the whole space of prices $\mathbb{P}$ - which includes vectors $(p, q)$ such that $p_{0}$ is zero - it may not hold even when preferences are strictly monotonic and physical endowments are interior points of the commodity space.
} 
EXAMPLE 3. We can include debt constraints that depend of consumption decisions. For instance, assume that there is $\kappa \in(0,1)$ such that, for any $(p, q) \in \mathbb{P}$ and for some $i \in \mathcal{I}$,

$$
\left(x^{i}, z^{i}\right) \in \Phi^{i}(p, q) \quad \Longrightarrow \quad \kappa p_{s} \cdot\left(w_{s}^{i}+Y_{s} x_{0}^{i}\right)+\sum_{j \in \mathcal{J}} R_{s, j}(p) \min \left\{z_{j}^{i}, 0\right\} \geq 0, \forall s \in S
$$

where $\sum_{s \in S} R_{s, j}(p)>0$ for each $j \in \mathcal{J}$. Then, agent $i$ 's debt is not greater than an exogenouslyfixed portion $\kappa$ of her physical-resources' value. If a portion $\rho>\kappa$ of physical resources can be garnished in case of bankruptcy, the above restriction ensures that $i$ honors her commitments.

\section{BAsic Assumptions}

The following are the basic hypotheses over fundamentals:

\section{Assumption (A1)}

(i) For any $i \in \mathcal{I}, V^{i}$ is continuous, strictly quasi-concave, and locally non-satiated at each $s \in \mathcal{S} .^{5}$

(ii) One of following hypotheses hold:

(a) For each $(s, l) \in \mathcal{S} \times \mathcal{L}$, there exists $i \in \mathcal{I}$ such that $V^{i}$ is strictly increasing in $x_{s, l}$. In addition, $W^{i} \gg 0, \forall i \in \mathcal{I}{ }^{6}$

(b) There exists $i \in \mathcal{I}$ such that, for each $(s, l) \in \mathcal{S} \times \mathcal{L}, V^{i}$ is strictly increasing in $x_{s, l}$. In addition, $W_{s}^{k} \neq 0, \forall(k, s) \in \mathcal{I} \times \mathcal{S}$ and $W^{i} \gg 0$.

(iii) Asset payments are continuous functions of prices satisfying $R_{j}(p) \neq 0, \forall j \in \mathcal{J}, \forall p \gg 0$.

\section{Assumption (A2)}

The correspondences $\left\{\Phi^{i}\right\}_{i \in \mathcal{I}}$ are lower hemicontinuous with convex values and closed graph relative to $\mathbb{P} \times \mathbb{E}$. In addition, agents are not burden to trade assets, i.e., $(0,0) \in \bigcap_{(p, q) \in \mathbb{P}} \Phi^{i}(p, q), \forall i \in \mathcal{I}$.

Under (A1)-(A2) individuals' choice set correspondences $\left\{C^{i}\right\}_{i \in \mathcal{I}}$ vary continuously with prices and, therefore, they do not compromise the continuity of individual demands (see Lemma 1).

\footnotetext{
${ }^{5}$ Strict quasi-concavity requires that, given $x^{i}, y^{i} \in \mathbb{R}_{+}^{\mathcal{L} \times \mathcal{S}}, V^{i}\left(\lambda x^{i}+(1-\lambda) y^{i}\right)>\min \left\{V^{i}\left(x^{i}\right), V^{i}\left(y^{i}\right)\right\}$ when $V^{i}\left(x^{i}\right) \neq V^{i}\left(y^{i}\right)$ and $\lambda \in(0,1)$. Local non-satiation at state of nature $s$ requires that, for each $\left(x_{k}^{i}\right)_{k \in \mathcal{S}} \in \mathbb{R}_{+}^{\mathcal{L} \times \mathcal{S}}$ and $\epsilon>0$ there exists $y_{s}^{i} \in \mathbb{R}_{+}^{\mathcal{L}}$ such that, $\left\|y_{s}^{i}-x_{s}^{i}\right\|<\epsilon$ and $V^{i}\left(\left(x_{k}^{i}\right)_{k \neq s}, y_{s}^{i}\right)>V^{i}\left(\left(x^{i}\right)_{k \in \mathcal{S}}\right)$.

${ }^{6}$ The interiority of physical endowments is a traditional assumption in models with heterogenous access to financial markets (see, for instance, Siconolfi (1989); Polemarchakis and Siconolfi (1997); Cass, Siconolfi and Villanacci (2001); Carosi, Gori and Villanaci (2009); Aouani and Cornet (2009, 2011); Cornet and Gopalan (2011)). Furthermore, it is not clear that (A2)(ii) can be relaxed without compromise the generality of our approach to credit segmentation. In fact, although Gottardi and Hens (1996) shown the existence of equilibria in incomplete markets when endowments lie in the boundary of the consumption set and preferences are strictly monotonic, their result requires that any subset of individuals can redistributed resources - through financial trade - to the complementary subset of individuals.
} 
When borrowing constraints are independent of prices and given by $\Phi^{i}(p, q)=\mathbb{R}_{+}^{\mathcal{L} \times \mathcal{S}} \times Z^{i}$, with $Z^{i}+\mathbb{R}_{+}^{\mathcal{J}} \subseteq Z^{i}$, Assumption (A2) holds if and only if $\left\{Z^{i}\right\}_{i \in \mathcal{I}}$ are closed and convex sets containing zero. Alternatively, if individuals are burden by price-dependent borrowing constraints, i.e.,

$$
\Phi^{i}(p, q):=\left\{\left(x^{i}, z^{i}\right) \in \mathbb{E}: z^{i}+g_{k}^{i}(p, q) \geq 0, \forall k \in\left\{1, \ldots, m_{i}\right\}\right\}, \quad \forall i \in \mathcal{I}
$$

then Assumption (A2) holds if and only if $g_{k}^{i}: \mathbb{P} \rightarrow \mathbb{R}$ is continuous for every $i \in \mathcal{I}$ and $1 \leq k \leq m_{i}$.

As in Seghir and Torres-Martínez (2011), we can include trading constraints determining restrictions on borrowing and first-period consumption,

$$
\Phi^{i}(p, q):=\left\{\left(x^{i}, z^{i}\right) \in \mathbb{E}: \exists\left(\theta^{i}, \varphi^{i}\right) \in \mathbb{R}_{+}^{\mathcal{J}} \times \mathbb{R}_{+}^{\mathcal{J}}, \varphi^{i} \in \Psi^{i}\left(x_{0}^{i}\right) \wedge z^{i}=\theta^{i}-\varphi^{i}\right\}
$$

where $\Psi^{i}: \mathbb{R}_{+}^{\mathcal{L}} \rightarrow \mathbb{R}_{+}^{\mathcal{J}}$. In this context, if $\left\{\Psi^{i}\right\}_{i \in \mathcal{I}}$ have a closed and convex graph and $0 \in \Psi^{i}\left(x_{0}^{i}\right)$ for each $x_{0}^{i} \in \mathbb{R}_{+}^{\mathcal{L}}$, then Assumption (A2) is satisfied. Furthermore, if $\Psi^{i}\left(x_{0}^{i}\right) \subseteq \Psi^{i}\left(y_{0}^{i}\right)$ for each $y_{0}^{i} \geq x_{0}^{i}$, then we can ensure that $\Phi^{i}(p, q)+\mathbb{R}_{+}^{\mathcal{L} \times \mathcal{S}} \times \mathbb{R}_{+}^{\mathcal{J}} \subseteq \Phi^{i}(p, q), \forall(p, q) \in \mathbb{P}$. We remark that our approach is complementary to Seghir and Torres-Martínez (2011), because at the cost of a non-redundancy assumption and a super-replication property (see Assumptions (A3) and (A4) below) we neither restrict individual preferences nor require $\left\{\Psi^{i}\right\}_{i \in \mathcal{I}}$ to have compact values.

\section{Equilibrium Existence}

We analyze the existence of a competitive equilibrium using standard fixed point techniques. For this reason, we want to ensure that endogenous variables can be bounded without affecting individual's behavior or excess of demand correspondences.

As was pointed out by Hart (1975), even without trading constraints, equilibrium may cease to exists when markets are incomplete, assets are real, and admissible short-sales are unbounded. For this reason, we induce upper bounds on individual variables through restrictions over the correspondence of attainable allocations $\Omega: \mathbb{P} \rightarrow \mathbb{E}^{\mathcal{I}}$, which is given by

$$
\Omega(p, q):=\left\{\left(\left(x^{i}, z^{i}\right)\right)_{i \in \mathcal{I}} \in \prod_{i \in \mathcal{I}} C^{i}(p, q): \sum_{i \in \mathcal{I}}\left(x^{i}, z^{i}\right)=\sum_{i \in \mathcal{I}}\left(W^{i}, 0\right)\right\} .
$$

\section{Assumption (A3)}

For any compact set $\mathbb{P}^{\prime} \subseteq \mathbb{P}, \bigcup_{(p, q) \in \mathbb{P}^{\prime}:(p, q) \gg 0} \Omega(p, q)$ is bounded.

Since market-feasible consumption allocations are bounded, the objective of (A3) is to guarantee the existence of bounds on market-feasible financial positions. Essentially, there are three independent factors which contribute to ensure that (A3) holds: the specification of trading constraints, the non-negativity of consumption, and the restrictions imposed by market feasibility. 
Thus, for instance, Assumption (A3) holds as a consequence of the specification of trading constraints and market feasibility when there are short-sale constraints: for some continuous function $m: \mathbb{P} \rightarrow \mathbb{R}_{+}^{\mathcal{J}}$ and for every $\left(x^{i}, z^{i}\right) \in \Phi^{i}(p, q), z^{i} \geq-m(p, q), \forall i \in \mathcal{I}, \forall(p, q) \in \mathbb{P}$. Alternatively, the non-negativity of consumption guarantees (A3) when $\mathcal{J}$ is composed by non-redundant nominal assets. Furthermore, if debts are backed by physical collateral, then market feasibility induces bounds on portfolios as a consequence of the scarcity of durable goods.

Notice that, Assumption (A3) is satisfied by trading constraints described in Example 1 and by any financial market composed by contracts as those described in Examples 2 and 3.

In the following result we illustrate how Assumption (A3) is related with a non-redundancy condition over the financial structure, which avoids the existence of unbounded sequences of trading admissible portfolios that do not generate transfers at the second period.

Proposition. Under (A1)(ii)(a) and (A2), Assumption (A3) holds when

$$
\bigcup_{(p, q) \in \mathbb{P}} \bigcup_{i \in \mathcal{I}}\left\{z^{i} \in \mathbb{R}^{\mathcal{J}} \backslash\{0\}: \sum_{j \in \mathcal{J}} R_{j}(p) z_{j}^{i}=0 \wedge\left(W^{i}, \delta z^{i}\right) \in \Phi^{i}(p, q), \forall \delta>0\right\}=\emptyset
$$

Condition (NR) is a generalization of the hypothesis of non-redundancy introduced by Siconolfi

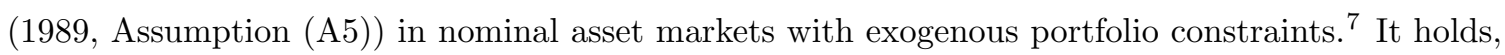
for instance, when $\left\{R_{j}(p)\right\}_{j \in \mathcal{J}}$ is linearly independent for every $(p, q) \in \mathbb{P}$. Hence, for instance, Assumption (A3) is satisfied in a market composed by just two assets, a risk free nominal contract and a real security making a promise $A \in \mathbb{R}_{++}^{\mathcal{L}}$ contingent to a state $s_{0} \in S$.

It is important to remark that, as the following example illustrates, even when assets are nominal and trading constraints are independent of prices, the non-redundancy condition (NR) is stronger than Assumption (A3).

EXAmple 4. Assume that financial markets are composed by two risk-free nominal assets which pay one unit of account at each state of nature in the second period. Also, there are two agents, $A$ and $B$, such that $\Phi^{A}(p, q)=\mathbb{R}_{+}^{\mathcal{L} \times \mathcal{S}} \times[-1,+\infty) \times \mathbb{R}$ and $\Phi^{B}(p, q)=\mathbb{R}_{+}^{\mathcal{L} \times \mathcal{S}} \times[-1,+\infty) \times[-1,+\infty)$.

\footnotetext{
${ }^{7}$ Indeed, assume that assets are nominal (i.e., for every $(s, j) \in S \times \mathcal{J}, R_{s, j}(p)=N_{s, j}$ ) and trading constraints are given by exogenous portfolio restrictions (i.e., for every $i \in \mathcal{I}$ there is $Z^{i} \subseteq \mathbb{R}^{\mathcal{J}}$ such that $\Phi^{i}(p, q)=\mathbb{R}_{+}^{\mathcal{L} \times \mathcal{S}} \times$ $\left.Z^{i}, \forall(p, q) \in \mathbb{P}\right)$. Then, (NR) is satisfied if and only if

$$
\bigcup_{i \in \mathcal{I}}\left\{z^{i} \in \mathbb{R}^{\mathcal{J}} \backslash\{0\}: N z^{i}=0 \wedge \delta z^{i} \in Z^{i}, \forall \delta>0\right\}=\emptyset
$$
}

where $N=\left(N_{s, j}\right)_{(s, j) \in S \times \mathcal{J}}$ is the matrix of asset payments. 
Then, (A3) holds, because for any compact set $\mathbb{P}^{\prime} \subseteq \mathbb{P}$ and for each $(p, q) \in \mathbb{P}^{\prime}$ we have that,

$$
\left(\left(x^{i}, z^{i}\right)\right)_{i \in\{A, B\}} \in \Omega(p, q) \quad \Longrightarrow \quad\left(x^{i}, z^{i}\right) \in[0, \alpha]^{\mathcal{L} \times \mathcal{S}} \times\left([-1,1] \times\left[-\beta\left(\mathbb{P}^{\prime}\right), \beta\left(\mathbb{P}^{\prime}\right)\right]\right), \quad \forall i \in\{A, B\},
$$

where

$$
\alpha=\sum_{(s, l) \in \mathcal{S} \times \mathcal{L}}\left(W_{s, l}^{A}+W_{s, l}^{B}\right), \quad \beta\left(\mathbb{P}^{\prime}\right)=1+\max _{(p, q) \in \mathbb{P}^{\prime}} \sum_{s \in S} p_{s}\left(W_{s}^{A}+W_{s}^{B}\right) .
$$

However, (NR) is not satisfied, because

$$
(1,-1) \in\left\{z^{A} \in \mathbb{R}^{2} \backslash\{0\}: z_{1}^{A}+z_{2}^{A}=0 \wedge\left(W^{A}, \delta z^{A}\right) \in \Phi^{A}(p, q), \forall \delta>0\right\} .
$$

To obtain upper bounds for prices, we restrict the heterogeneity of financial transfers through a super-replication property, requiring that wealth transfers that are implementable by long positions in segmented markets can be super-replicated by investments in non-segmented assets. Essentially, as prices of commodities and unsegmented assets can be normalized without induce frictions on individual behavior, the super-replication property allows us to find endogenous upper bounds for non-arbitrage prices of segmented assets (see Lemma 3 in the Appendix).

In order to formalize these ideas, we need a previous definition: a contract $j$ is unsegmented if for every vector of prices $(p, q) \in \mathbb{P}$ there exists $\delta>0$ such that $-\delta \vec{e}_{j} \in \bigcap_{i \in \mathcal{I}} \Phi^{i}(p, q)$, where $\vec{e}_{j} \in \mathbb{E}$ is the allocation composed by just one unit of $j$. Let $\mathcal{J}_{a}$ be the set of unsegmented contracts.

\section{Assumption (A4)}

(i) For each compact set $\mathbb{P}^{\prime} \subseteq \mathbb{P}$, there exists $\left(\widehat{x}_{0}, \widehat{z}\right) \in \mathbb{R}_{+}^{\mathcal{L}} \times \mathbb{R}_{+}^{\mathcal{J}_{a}}$ such that,

$$
\sum_{j \in \mathcal{J} \backslash \mathcal{J}_{a}} R_{s, j}(p) \leq p_{s} \cdot Y_{s} \widehat{x}_{0}+\sum_{k \in \mathcal{J}_{a}} R_{s, k}(p) \widehat{z}_{k}, \quad \forall s \in S, \forall(p, q) \in \mathbb{P}^{\prime} .
$$

(ii) Long-positions in contracts $\mathcal{J} \backslash \mathcal{J}_{a}$ can be reduced without compromise trading admissibility. ${ }^{8}$

Notice that $\mathcal{J} \backslash \mathcal{J}_{a}$ is the set of segmented credit contracts. Thus, Assumption (A4)(i) requires that payments associated to segmented contracts can be super-replicated by positions on durable goods and/or contracts that all agents can short-sale. In particular, the set of states of nature where inter-temporal transfers are available is the same for all agents. Furthermore, when (A4)(ii) holds, segmented contracts are not required as financial guarantees to obtain access to credit.

Our main result ensures that credit segmentation is compatible with equilibria:

THEOREM. Under Assumptions (A1)-(A4) there is a competitive equilibrium.

\footnotetext{
${ }^{8}$ That is, given $\left(x^{i}, z^{i}\right) \in \Phi^{i}(p, q)$ and $\pi \in[0,1)$, if $z_{j}^{i}>0$ for some $j \notin \mathcal{J}_{a}$, then $\left(x^{i},\left(z_{k}^{i}\right)_{k \neq j}, \pi z_{j}^{i}\right) \in \Phi^{i}(p, q)$.
} 
We want to illustrate several situations where the super-replication property holds.

In the following result we assume that there is an asset that all agents can short-sale and whose payments are positive whenever one of the remaining contracts has non-trivial promises. Note that, this is satisfied when all agents have access to borrowing through a risk-free asset.

Corollary 1. Under Assumptions (A1)-(A3) and (A4)(ii), assume that there is an unsegmented contract $j \in \mathcal{J}_{a}$ such that $\left(R_{s, j}(p)\right)_{s \in S_{j}} \gg 0, \forall(p, q) \in \mathbb{P}$, where $S_{j}:=\left\{s \in S: \exists k \neq j, R_{s, k} \neq 0\right\}$. Then, a competitive equilibrium always exists.

The super-replication property also holds when assets' promises are measure in units of nonperishable commodities. Indeed, if we define $\mathcal{L}^{*}:=\left\{l \in L: Y_{s}(l, l)>0, \forall s \in \bigcup_{j} S_{j}\right\}$ as the set of commodities that not-fully depreciate at the states of nature where financial contracts make non-trivial promises, we have that:

Corollary 2. Under Assumptions (A1)-(A3) and (A4)(ii), there is a competitive equilibrium if assets are real and promises are given in terms of commodities in $\mathcal{L}^{*}$.

Our model is general enough to be compatible with the inclusion of default and non-recourse collateralized assets. That is, assets whose promises are backed by non-perishable commodities such that the only payment enforcement mechanism in case of default is given by the seizure of these guarantees. ${ }^{9}$ In this direction, we can extend the model of Geanakoplos and Zame (2013) to include financial market segmentation and price-dependent trading constraints.

More precisely, assume that each asset $j \in \mathcal{J}$ is characterized by a pair $\left(C_{j},\left(D_{s, j}(p)\right)_{s \in S}\right)$, where $C_{j}=\left(C_{j, l}\right)_{l \in \mathcal{L}} \in \mathbb{R}_{+}^{\mathcal{L}} \backslash\{0\}$ is the collateral guarantee and $\left(D_{s, j}(p)\right)_{s \in S} \in \mathbb{R}_{+}^{S}$ are the state contingent promises. Borrowers are required to pledge the associated collateral, i.e., for any $\left(x^{i}, z^{i}\right) \in \Phi^{i}(p, q)$ we have that $x_{0}^{i} \geq \sum_{j \in \mathcal{J}} C_{j} \max \left\{-z_{j}^{i}, 0\right\}$. In addition, as the only enforcement in case of default is the seizure of collateral guarantees, borrowers give strategic default, delivering the minimum between collateral value and promises, i.e., $R_{s, j}(p):=\min \left\{D_{s, j}(p), p_{s} Y_{s} C_{j}\right\}, \forall s \in S$.

Notice that, payments associated to a non-recourse collateralized loan can be super-replicated by the bundle of commodities used as guarantee. Hence, non-arbitrage asset prices can be bounded from above by the collateral cost (see Appendix).

\footnotetext{
${ }^{9}$ In the absence of payment enforcement mechanisms over collateral repossession, the non-satiability of preferences guarantees that borrowers of a collateralized loan always deliver the minimum between promises and collateral values. Therefore, lenders that finance these loans perfectly foresight the payments that they will receive. Hence, as in Geanakoplos and Zame (2013), we can capture with a same financial contract both the collateralized line of credit and the collateralized loan obligation (CLO) that passthrough the payments made by borrowers.
} 
Corollary 3. Under Assumptions (A1)-(A3) and (A4)(ii) there exists a competitive equilibria in collateralized asset markets.

Since Corollaries 2 and 3 do not require $\mathcal{J}_{a} \neq \emptyset$, they are compatible with an extreme form of financial segmentation: the exclusion of some agents from credit markets.

We conclude this section with some examples which highlight the difficulties to guarantee equilibrium existence when our assumptions are not satisfied. In the first one, we present an economy without uncertainty where a competitive equilibrium cease to exists because both the super-replication property and Assumption (A1)(ii) are not satisfied. In the second example, inspired by Hahn and Won (2007, Example 5.1), we describe an economy where there do not exist equilibria because Assumption (A4) does not holds.

EXAMPLE 5. Consider a two-period economy without uncertainty at the second period. There is a perishable commodity, used as the numeraire, and a real asset $j$ that promises to deliver one unit of commodity at the second period. There are two agents, $A$ and $B$, which are characterized by utility functions $V^{A}\left(x_{0}, x_{1}\right)=V^{B}\left(x_{0}, x_{1}\right)=\sqrt{x_{0}}+0.5 \sqrt{x_{1}}$ and by endowments $\left(w^{A}, w^{B}\right)=((1,1),(1,0))$. In addition, $\Phi^{A}(p, q)=\mathbb{R}_{+}^{2} \times \mathbb{R}_{+}$and $\Phi^{B}(p, q)=\mathbb{R}_{+}^{2} \times \mathbb{R}$. Hence, the real asset is segmented, because only agent $B$ can short-sale it.

It follows that Assumptions (A1)(i), (A1)(iii), (A2), (A3), and (A4)(ii) hold. However, since the commodity is perishable and asset $j$ is segmented, the super-replication property (A4)(i) is not satisfied. Also, (A1)(ii) does not hold, because $w^{B}=(1,0)$.

The failure of these assumptions implies that the economy does not have equilibria. Indeed, by monotonicity of preferences the asset price $q$ is strictly positive. Hence, Inada's condition and budget feasibility imply that the optimal portfolio of agent $B$ belongs to $(0,1 / q]$. This is incompatible with credit segmentation, because only $B$ can short-sale $j .{ }^{10}$

${ }^{10}$ If $\Phi^{A}(p, q)=\mathbb{R}_{+}^{2} \times[-\delta,+\infty)$, for some $\delta>0$, then the super-replication property (A4)(i) holds. Moreover, when $\delta \neq 2 /(4+\sqrt{2})$, there is a unique competitive equilibrium, which is given by

$$
\left(q,\left(x_{0}^{A}, x_{1}^{A}, z^{A}\right),\left(x_{0}^{B}, x_{1}^{B}, z^{B}\right)\right)=\left(\frac{\sqrt{2}}{2},\left(1+\frac{\sqrt{2}}{4+\sqrt{2}}, 1-\frac{2}{4+\sqrt{2}},-\frac{2}{4+\sqrt{2}}\right),\left(1-\frac{\sqrt{2}}{4+\sqrt{2}}, \frac{2}{4+\sqrt{2}}, \frac{2}{4+\sqrt{2}}\right)\right)
$$

for $\delta>2 /(4+\sqrt{2}) ;$ and by

$$
\left(q,\left(x_{0}^{A}, x_{1}^{A}, z^{A}\right),\left(x_{0}^{B}, x_{1}^{B}, z^{B}\right)\right)=\left(\frac{1}{8}\left(\sqrt{1+\frac{16}{\delta}}-1\right),(1+q \delta, 1-\delta,-\delta),(1-q \delta, \delta, \delta)\right)
$$

for $\delta<2 /(4+\sqrt{2})$. When $\delta=2 /(4+\sqrt{2})$ these two combination of prices and allocations are equilibria. Notice that, the equilibrium asset price diverges as $\delta$ goes to zero. 
ExAmple 6. Consider an economy $\mathcal{E}$ with two states of nature at the second period $S=\{u, d\}$. There is only one commodity, which is perishable. Two agents, $A$ and $B$, that only demand the commodity at the second period, ${ }^{11}$ are characterized by the following preferences and endowments:

$$
\begin{array}{ll}
V^{A}\left(x_{u}, x_{d}\right)=2 \sqrt{x_{u}+1}+\sqrt{x_{d}}, & \left(w_{u}^{A}, w_{d}^{A}\right)=(1,1) ; \\
V^{B}\left(x_{u}, x_{d}\right)=\sqrt{x_{u}+1}+2 \sqrt{x_{d}}, & \left(w_{u}^{B}, w_{d}^{B}\right)=(1,1) .
\end{array}
$$

There are two segmented Arrow securities satisfying $R_{1}(p)=\left(p_{u}, 0\right), R_{2}(p)=\left(0, p_{d}\right)$. We assume that, for agent $i, \Phi^{i}(p, q):=\left\{\left(\left(x_{u}^{i}, x_{d}^{i}\right),\left(z_{1}^{i}, z_{2}^{i}\right)\right) \in \mathbb{R}_{+}^{2} \times \mathbb{R}^{2}: z_{1}^{i}+z_{2}^{i} \geq 0\right\}$.

It is not difficult to verify that Assumptions (A1)-(A3) are satisfied. However, as trading constraints requires that $z_{1}^{i}+z_{2}^{i} \geq 0$, the set of unsegmented assets in empty and long-position on segmented Arrow securities cannot be reduced without compromise its financial admissibility. Hence, Assumption (A4) does not holds.

We affirm that $\mathcal{E}$ does not have equilibria.

If there is a competitive equilibrium, the strict monotonicity of preferences ensures that commodity and asset prices are strictly positive. Hence, at each state of nature in the second period, we can use the commodity as numeraire. By contradiction, assume that there is an equilibrium $\left(\bar{q}_{1}, \bar{q}_{2},\left(\left(\bar{x}_{u}^{i}, \bar{x}_{d}^{i}\right),\left(\bar{z}_{1}^{i}, \bar{z}_{2}^{i}\right)\right)_{i \in\{A, B\}}\right)$. Since trading constraints require that $\bar{z}_{1}^{i}+\bar{z}_{2}^{i} \geq 0$, market feasibility guarantees that $\bar{\alpha}:=\bar{z}_{1}^{A}=-\bar{z}_{2}^{A}=\bar{z}_{2}^{B}=-\bar{z}_{1}^{B}$. Therefore, the strict monotonicity of preferences implies that $\left(\bar{x}_{u}^{A}, \bar{x}_{d}^{A}\right)=(1+\bar{\alpha}, 1-\bar{\alpha})$ and $\left(\bar{x}_{u}^{B}, \bar{x}_{d}^{B}\right)=(1-\bar{\alpha}, 1+\bar{\alpha})$.

It follows that $\bar{\alpha}$ is the unique element in the following sets:

$$
\underset{-1,1]:\left(_{(1}-\bar{q}_{2}\right) \alpha=0}{\operatorname{argmax}} 2 \sqrt{2+\alpha}+\sqrt{1-\alpha}, \quad \underset{\alpha \in[-1,1]:\left(\bar{q}_{1}-\bar{q}_{2}\right) \alpha=0}{\operatorname{argmax}} \sqrt{2-\alpha}+2 \sqrt{1+\alpha} .
$$

Therefore, we have two cases. If $\bar{q}_{1}=\bar{q}_{2}$, then Inada's condition implies that $\bar{\alpha} \in(-1,1)$, while the first order conditions ensures that $\sqrt{2+\bar{\alpha}}=2 \sqrt{1-\bar{\alpha}}$ and $2 \sqrt{2-\bar{\alpha}}=\sqrt{1+\bar{\alpha}}$. However, this system of equations does not have a solution. Alternatively, if $\bar{q}_{1} \neq \bar{q}_{2}$, then $\bar{\alpha}=0$ and the first orden conditions of problems above induce a contradiction as $\sqrt{2} \neq 2$.

\section{Concluding Remarks}

In this paper we extend the theory of general equilibrium with incomplete financial markets to include price-dependent trading constraints that restrict both consumption alternatives and credit access. Our approach is general enough to incorporate several types of dependencies between prices,

\footnotetext{
${ }^{11}$ Our model can be easily modified to include economies where agents only trade assets at $t=0$. In fact, it is sufficient to allow the sets of commodities to depend on the state of nature $\left(\mathcal{L}_{s}\right)_{s \in \mathcal{S}}$, making $\mathcal{L}_{0}=\emptyset$. In this context, the existence of a competitive equilibrium can be obtained by identical arguments to those made in the proof of our main Theorem. Of course, Assumption (A1)(ii) changes because agents do not have physical endowments at $t=0$. In addition, in the statement of the super-replication property the bundle $\widehat{x}_{0}$ disappears.
} 
consumption, and credit access. For instance, the access to liquidity may depend on individuals income, the short-sale of derivatives may require the deposit of margins, or borrowers could be required to pledge physical collateral to protect lenders in case of default.

As we want to include financial segmentation, our results of equilibrium existence do not rely on financial survival conditions. Thus, based on the idea that asset prices can be bounded when their promises can be super-replicated at a finite cost, we show equilibrium existence assuming that investments in non-segmented assets can super-replicate the deliveries of segmented contracts and that investment in segmented contracts is not required to obtain access to credit.

In our model, investment restrictions can be introduced at the cost of more notation and technical hypotheses (see Cea-Echenique and Torres-Martínez (2014)). As a matter of future research, we want to analyze the characteristics of trading constraints that are essential to guarantee equilibrium existence in economies with more than two periods (cf., Iraola and Torres-Martínez (2014)).

\section{Appendix}

\section{Proof of the Proposition.}

Under (A1)(ii)(a) and (A2), suppose that (NR) holds. By contradiction, assume that there is a compact set $\mathbb{P}^{\prime} \subseteq \mathbb{P}$ such that $\bigcup_{(p, q) \in \mathbb{P}^{\prime}:(p, q) \gg 0} \Omega(p, q)$ is unbounded. Hence, there is an unbounded sequence $\left\{\left(\left(p_{n}, q_{n}\right),\left(x_{n}^{i}, z_{n}^{i}\right)_{i \in \mathcal{I}}\right)\right\}_{n \in \mathbb{N}} \subseteq \mathbb{P}^{\prime} \times \mathbb{E}^{\mathcal{I}}$ for which $\left(x_{n}^{i}, z_{n}^{i}\right) \in \Omega\left(p_{n}, q_{n}\right), \forall n \in \mathbb{N}$. It follows that, for every $n$ and $i,\left(W, z_{n}^{i}\right) \in \Phi^{i}\left(p_{n}, q_{n}\right)$, where $W=\left(W_{s}\right)_{s \in \mathcal{S}}:=\left(\sum_{i \in \mathcal{I}} W_{s}^{i}\right)_{s \in S}$. In particular, for some agent $h$ there is an unbounded subsequence $\left\{z_{n_{k}}^{h}\right\}_{k \in \mathbb{N}} \subseteq\left\{z_{n}^{h}\right\}_{n \in \mathbb{N}}$ such that, for every $k \in \mathbb{N}, z_{n_{k}}^{h} \neq 0,\left\|z_{n_{k}}^{h}\right\|_{\Sigma} \leq\left\|z_{n_{k+1}}^{h}\right\|_{\Sigma}$, and $\left(W, z_{n_{k}}^{h}\right) \in \Phi^{h}\left(p_{n_{k}}, q_{n_{k}}\right)$.

Let $\left((\tilde{p}, \tilde{q}), \tilde{z}^{h}\right)$ be a cluster point of $\left\{\left(p_{n_{k}}, q_{n_{k}}\right), z_{n_{k}}^{h} /\left\|z_{n_{k}}^{h}\right\|_{\Sigma}\right\}_{k \in \mathbb{N}}$. We want to contradict (NR) by showing that $\tilde{z}^{h} \neq 0$ belongs to $\left\{z^{h} \in \mathbb{R}^{\mathcal{J}}: \sum_{j \in \mathcal{J}} R_{j}(\tilde{p}) z_{j}^{h}=0 \wedge\left(W^{h}, \delta z^{h}\right) \in \Phi^{h}(\tilde{p}, \tilde{q}), \forall \delta>0\right\}$.

We affirm that $\sum_{j \in \mathcal{J}} R_{s, j}(\tilde{p}) \tilde{z}_{j}^{h}=0, \forall s \in S$. First, if there is $s \in S$ such that $\sum_{j \in \mathcal{J}} R_{s, j}(\tilde{p}) \tilde{z}_{j}^{h}<0$, then the compactness of $\mathbb{P}^{\prime}$ ensures that there is $a>0$ such that $a \sum_{j \in \mathcal{J}} R_{s, j}(\tilde{p}) \tilde{z}_{j}^{h}<-2 \tilde{p}_{s} \cdot W_{s}$. This implies that, for $k \in \mathbb{N}$ large enough, $a \sum_{j \in \mathcal{J}} R_{s, j}\left(p_{n_{k}}\right) z_{n_{k}, j}^{h} /\left\|z_{n_{k}}^{h}\right\|_{\Sigma}<-2 p_{n_{k}, s} \cdot W_{s}$. Since $\lim _{k}\left\|z_{n_{k}}^{h}\right\|_{\Sigma}=+\infty$, it follows that for $k$ large enough $\sum_{j \in \mathcal{J}} R_{s, j}\left(p_{n_{k}}\right) z_{n_{k}, j}^{h}<-2 p_{n_{k}, s} \cdot W_{s}$, a contradiction with $\left(x_{n_{k}}^{i}, z_{n_{k}}^{i}\right)_{i \in \mathcal{I}} \in$ $\Omega\left(p_{n_{k}}, q_{n_{k}}\right)$. Second, if there is $s \in S$ such that $\sum_{j \in \mathcal{J}} R_{s, j}(\tilde{p}) \tilde{z}_{j}^{h}>0$, then $b \sum_{j \in \mathcal{J}} R_{s, j}(\tilde{p}) \tilde{z}_{j}^{h}>2(\# \mathcal{I}-1) \tilde{p}_{s}$. $W_{s}$, for some $b>0$. Hence, for $k \in \mathbb{N}$ large enough, we have that $\sum_{j \in \mathcal{J}} R_{s, j}\left(p_{n_{k}}\right) z_{n_{k}, j}^{h}>2(\# \mathcal{I}-1) p_{n_{k}, s} \cdot W_{s}$. Due to $\sum_{i \in \mathcal{I}} \sum_{j \in \mathcal{J}} R_{s, j}\left(p_{n_{k}}\right) z_{n_{k}, j}^{i}=0$, there exists $h^{\prime} \neq h$ such that $\sum_{j \in \mathcal{J}} R_{s, j}\left(\tilde{p}_{n_{k}}\right) z_{n_{k}, j}^{h^{\prime}}<-2 \tilde{p}_{n_{k}, s} \cdot W_{s}$, a contradiction with $\left(x_{n_{k}}^{i}, z_{n_{k}}^{i}\right)_{i \in \mathcal{I}} \in \Omega\left(p_{n_{k}}, q_{n_{k}}\right)$.

Given $\delta>0$, we affirm that $\left(W^{h}, \delta \tilde{z}^{h}\right) \in \Phi^{h}(\tilde{p}, \tilde{q})$. Indeed, given $c>0$ there exists $k_{c} \in \mathbb{N}$ such that $\left\|z_{n_{k}}^{h}\right\|_{\Sigma} \geq c, \forall k \geq k_{c}$. Hence, as $\Phi^{h}$ has convex values and $(W, 0) \in \Phi^{h}\left(p_{n_{k}}, q_{n_{k}}\right)$ for every $k \in \mathbb{N}$, it follows that $\left(1-c /\left\|z_{n_{k}}^{h}\right\|_{\Sigma}\right)(W, 0)+c /\left\|z_{n_{k}}^{h}\right\|_{\Sigma}\left(W, z_{n_{k}}^{h}\right)=\left(W, c z_{n_{k}}^{h} /\left\|z_{n_{k}}^{h}\right\|_{\Sigma}\right) \in \Phi^{h}\left(p_{n_{k}}, q_{n_{k}}\right)$, for any $k \geq k(\delta)$, which in turn implies that $\left(W, c \tilde{z}^{h}\right) \in \Phi^{h}(\tilde{p}, \tilde{q})$. Furthermore, Assumption (A1)(ii)(a) guarantees that there is $\sigma \in(0,1)$ such that, $\sigma W_{s} \ll W_{s}^{h}, \forall s \in S$. Since $(1-\sigma)(0,0)+\sigma\left(W,(\delta / \sigma) \tilde{z}^{h}\right) \in \Phi^{h}(p, q)$, we conclude that $\left(W^{h}, \delta \tilde{z}^{h}\right) \in \Phi^{h}(\tilde{p}, \tilde{q})$, because $\Phi^{h}(\tilde{p}, \tilde{q})+\mathbb{R}_{+}^{\mathcal{L} \times \mathcal{S}} \times \mathbb{R}_{+}^{\mathcal{J}} \subseteq \Phi^{h}(\tilde{p}, \tilde{q})$. 
Proof of The TheOrem.

CASE I. Assume that (A1)(ii)(a) holds.

Given $M \in \mathbb{N}$, consider the normalized space of prices $\mathbb{P}(M):=\mathcal{P}_{0} \times[0, M]^{\mathcal{J}_{b}} \times \mathcal{P}_{1}^{S}$, where $\mathcal{P}_{0}:=\{y \in$ $\left.\mathbb{R}_{+}^{\mathcal{L} \cup \mathcal{J}_{a}}:\|y\|_{\Sigma}=1\right\}, \mathcal{P}_{1}:=\left\{y \in \mathbb{R}_{+}^{\mathcal{L}}:\|y\|_{\Sigma}=1\right\}$, and $\mathcal{J}_{b}:=\mathcal{J} \backslash \mathcal{J}_{a} \cdot{ }^{12}$ Note that, a typical element of $\mathbb{P}(M)$ is of the form $(p, q)=\left(\left(p_{0},\left(q_{k}\right)_{k \in \mathcal{J}_{a}}\right),\left(q_{j}\right)_{j \in \mathcal{J}_{b}},\left(p_{s}\right)_{s \in S}\right)$. When $(p, q) \in \mathbb{P}(M)$, the commodity price $p=\left(p_{0},\left(p_{s}\right)_{s \in S}\right)$ belongs to $\mathcal{P}:=\left\{y \in \mathbb{R}_{+}^{\mathcal{L}}:\|y\|_{\Sigma} \leq 1\right\} \times \mathcal{P}_{1}^{S}$.

Lemma 1. Under Assumptions (A1)(ii)-(iii) and (A2), for every agent $i \in \mathcal{I}$ the choice set correspondence $C^{i}: \mathbb{P}(M) \rightarrow \mathbb{E}$ is lower hemicontinuous with closed graph and non-empty and convex values.

Proof. Fix $i \in \mathcal{I}$. Since for every $(p, q) \in \mathbb{P}$ the allocation $\left(\left(W_{s}^{i}\right)_{s \in \mathcal{S}}, 0\right) \in C^{i}(p, q), C^{i}$ is non-empty valued. Assumption (A2) implies that $C^{i}$ has convex values and closed graph. To prove that $C^{i}$ is lower hemicontinuous, let $\stackrel{\circ}{C}^{i}: \mathbb{P}(M) \rightarrow \mathbb{E}$ be the correspondence that associates to each $(p, q) \in \mathbb{P}(M)$ the set of allocations $\left(x^{i}, z^{i}\right) \in C^{i}(p, q)$ satisfying budget constraints with strict inequalities. We affirm that $\dot{C}^{i}$ is lower hemicontinuous and has non-empty values. Since $C^{i}$ is the closure of $\dot{C}^{i}$, these properties imply that $C^{i}$ is lower hemicontinuous (see Border $(1985,11.19(\mathrm{c}))$ ). Thus, to obtain the results it is sufficient to ensure the claimed properties for $\stackrel{\circ}{C}^{i}$.

Claim A. $\stackrel{\circ}{C}^{i}$ has non-empty values. It follows from Assumption (A2) that $\left(\left(W_{0}^{i},\left(0.5 W_{s}^{i}\right)_{s \in S}\right), 0\right) \in \Phi^{i}(p, q)$ for all $(p, q) \in \mathbb{P}(M)$. Since $W^{i} \gg 0$, when $p_{0} \neq 0$ we always have that $\left(\left(W_{0}^{i},\left(0.5 W_{s}^{i}\right)_{s \in S}\right), 0\right) \in \dot{C}^{i}(p, q)$.

Thus, assume that $\mathcal{J}_{a} \neq \emptyset$ and fix $(p, q) \in \mathbb{P}(M)$ such that $p_{0}=0$ and, therefore $\left(q_{j}\right)_{j \in \mathcal{J}_{a}} \neq 0$. By definition of unsegmented contracts, for every $j \in \mathcal{J}_{a}$ there exists $\delta_{j}(p, q) \in(0,1)$ such that $-\delta_{j} \vec{e}_{j} \in \Phi^{i}(p, q)$ for all $\delta_{j} \in\left(0, \delta_{j}(p, q)\right)$. Since $\Phi^{i}$ has convex values, we conclude that there exists $\delta(p, q)>0$ such that $\left(\left(W_{0}^{i},\left(0.5 W_{s}^{i}\right)_{s \in S}\right), 0\right)-\delta \sum_{j \in \mathcal{J}_{a}} \vec{e}_{j} \in \Phi^{i}(p, q)$, for every $\delta \in(0, \delta(p, q)) .{ }^{13}$ Furthermore, assume that $\delta \in$ $(0, \delta(p, q))$ satisfies

$$
\delta \sum_{k \in \mathcal{J}_{a}} \max _{(\tilde{p}, s) \in \mathcal{P} \times S} R_{s, k}(\tilde{p})<0.5 \min _{(s, l) \in S \times \mathcal{L}} W_{s, l}^{i}
$$

Then, promises can be honored with the resources that became available after the consumption of $0.5 W_{s}^{i}$. Therefore, under these requirements, we have that $\left(\left(W_{0}^{i},\left(0.5 W_{s}^{i}\right)_{s \in S}\right), 0\right)-\delta \sum_{j \in \mathcal{J}_{a}} \vec{e}_{j} \in \stackrel{\circ}{C}^{i}(p, q)$.

Claim B. $\stackrel{\circ}{C}^{i}$ is lower hemicontinuous. Fix $(p, q) \in \mathbb{P}(M)$ and $\left(x^{i}, z^{i}\right) \in \check{C}^{i}(p, q)$. Given a sequence $\left\{\left(p_{n}, q_{n}\right)\right\}_{n \in \mathbb{N}} \subset \mathbb{P}(M)$ converging to $(p, q)$, the lower hemicontinuity of $\Phi^{i}$ (Assumption (A2)) ensures that there is $\left\{\left(x^{i}(n), z^{i}(n)\right)\right\}_{n \in \mathbb{N}} \subset \mathbb{E}$ converging to $\left(x^{i}, z^{i}\right)$ such that $\left(x^{i}(n), z^{i}(n)\right) \in \Phi^{i}\left(p_{n}, q_{n}\right), \forall n \in \mathbb{N}$. Thus, for $n \in \mathbb{N}$ large enough, $\left(x^{i}(n), z^{i}(n)\right) \in \stackrel{C}{C}^{i}\left(p_{n}, q_{n}\right)$. It follows from the sequential characterization of hemicontinuity that $\stackrel{\circ}{C}^{i}$ is lower hemicontinuous (see Border $(1985,11.11(\mathrm{~b}))$ ).

\footnotetext{
${ }^{12}$ Trading constraints are not necessarily homogeneous of degree zero in prices. Consequently, the normalization of prices may induce a selection of equilibria.

${ }^{13}$ It is sufficient to consider $\delta(p, q):=\min _{j \in \mathcal{J}_{a}} \delta_{j}(p, q) / \# \mathcal{J}_{a}$.
} 
It follows from Assumption (A3) that, when prices belongs to $\mathbb{P}(M)$ and are strictly positive, there is a finite upper bound for admissible financial positions:

$$
\bar{\Omega}(M):=2 \sup _{(p, q) \in \mathbb{P}(M):(p, q) \gg 0} \sup _{\left(x^{i}, z^{i}\right)_{i \in \mathcal{I}} \in \Omega(p, q)} \sum_{i \in \mathcal{I}}\left\|z^{i}\right\|_{\Sigma} .
$$

Given $(p, q) \in \mathbb{P}(M)$, for any $i \in \mathcal{I}$ we consider the truncated choice set $C^{i}(p, q) \cap \mathbb{K}(M)$, where

$$
\begin{gathered}
\mathbb{K}(M):=[0,2 \bar{W}(M)]^{\mathcal{L} \times \mathcal{S}} \times[-\bar{\Omega}(M), \# \mathcal{I} \bar{\Omega}(M)]^{\mathcal{J}}, \\
\bar{W}(M):=\left(\# J \# \mathcal{I} \bar{\Omega}(M)+\sum_{(s, l) \in \mathcal{S} \times \mathcal{L}} \sum_{i \in \mathcal{I}} W_{s, l}^{i}\right)\left(1+\max _{(p, s) \in \mathcal{P} \times S} \sum_{j \in \mathcal{J}} R_{s, j}(p)\right) .
\end{gathered}
$$

Let $\Psi_{M}: \mathbb{P}(M) \times \mathbb{K}^{\mathcal{I}}(M) \rightarrow \mathbb{P}(M) \times \mathbb{K}^{\mathcal{I}}(M)$ be the correspondence given by

$$
\Psi_{M}\left(p, q,\left(x^{i}, z^{i}\right)_{i \in \mathcal{I}}\right)=\phi_{0, M}\left(\left(x_{0}^{i}, z^{i}\right)_{i \in \mathcal{I}}\right) \times \prod_{s \in S} \phi_{s}\left(\left(x_{s}^{i}\right)_{i \in \mathcal{I}}\right) \times \prod_{i \in \mathcal{I}} \phi_{M}^{i}(p, q)
$$

where

$$
\begin{aligned}
\phi_{0, M}\left(\left(x_{0}^{i}, z^{i}\right)_{i \in \mathcal{I}}\right) & :=\underset{\left(p_{0}, q\right) \in \mathcal{P}_{0} \times[0, M] \mathcal{J}_{b}}{\operatorname{argmax}} p_{0} \cdot \sum_{i \in \mathcal{I}}\left(x_{0}^{i}-w_{0}^{i}\right)+q \cdot \sum_{i \in \mathcal{I}} z^{i} ; \\
\phi_{s}\left(\left(x_{s}^{i}\right)_{i \in \mathcal{I})}\right. & :=\underset{p_{s} \in \mathcal{P}_{1}}{\operatorname{argmax}} p_{s} \cdot \sum_{i \in \mathcal{I}}\left(x_{s}^{i}-W_{s}^{i}\right), \quad \forall s \in S ; \\
\phi_{M}^{i}(p, q) & :=\underset{\left(x^{i}, z^{i}\right) \in C^{i}(p, q) \cap \mathbb{K}(M)}{\operatorname{argmax}} V^{i}\left(x^{i}\right), \quad \forall i \in \mathcal{I} .
\end{aligned}
$$

Lemma 2. Under Assumptions (A1)-(A3), $\Psi_{M}$ has a non-empty set of fixed points.

Proof. By Kakutani's Fixed Point Theorem, it is sufficient to to prove that $\Psi_{M}$ has a closed graph with non-empty and convex values. Since $\mathbb{P}(M)$ is non-empty, convex and compact, Berge's Maximum Theorem establishes that $\left\{\phi_{0, M},\left\{\phi_{s}\right\}_{s \in S}\right\}$ have a closed graph with non-empty and convex values.

It remains to prove that the same properties hold for $\left\{\phi_{M}^{i}\right\}_{i \in \mathcal{I}}$. Given $i \in \mathcal{I}$, Lemma 1 implies that $C^{i}$ has a closed graph with non-empty and convex values. Since $\mathbb{K}(M)$ is compact and convex and $\left(\left(W_{s}^{i}\right)_{s \in \mathcal{S}}, 0\right) \in \mathbb{K}(M)$, it follows that $(p, q) \in \mathbb{P}(M) \rightarrow C^{i}(p, q) \cap \mathbb{K}(M)$ has a closed graph and non-empty, compact, and convex values. The proof of Lemma 1 also ensures that $C^{i}$ is lower hemicontinuous and $\left(\left(W_{s}^{i}\right)_{s \in \mathcal{S}}, 0\right) \in C^{i}(p, q) \cap \operatorname{int}(\mathbb{K}(M))$, where $\operatorname{int}(\mathbb{K}(M))$ denotes the interior of $\mathbb{K}(M)$ relative to $\mathbb{R}_{+}^{\mathcal{L} \times \mathcal{S}} \times \mathbb{R}^{\mathcal{J}}$. As $(p, q) \in \mathbb{P}(M) \rightarrow \operatorname{int}(\mathbb{K}(M))$ has open graph, it follows that $(p, q) \in \mathbb{P}(M) \rightarrow C^{i}(p, q) \cap \operatorname{int}(\mathbb{K}(M))$ is lower hemicontinuous (see Border $(1985,11.21(\mathrm{c}))$ ). Therefore, $(p, q) \in \mathbb{P}(M) \rightarrow C^{i}(p, q) \cap \mathbb{K}(M)$ is lower hemicontinuous too (see Border $(1985,11.19(\mathrm{c}))$ ). Berge's Maximum Theorem and the continuity and quasiconcavity of $V^{i}$ guarantees that $\phi_{M}^{i}$ satisfies the required properties.

Since Assumption (A4) holds, there exists $\left(\widehat{x}_{0}, \widehat{z}\right)$ that super-replicates the financial payments of segmented contracts when prices are in the compact set $\mathbb{P}(M)$. Notice that $\left(\widehat{x}_{0}, \widehat{z}\right)$ is independent of $M$, because financial payments are independent of asset prices. Let $\bar{Q}:=\max \left\{1,2\left(\left\|\widehat{x}_{0}\right\|_{\Sigma}+\max _{k \in \mathcal{J}_{a}} \widehat{z}_{k}\right)\right\}$. 
Lemma 3. Under (A1)(i), (A1)(iii) and (A4), let $\left(\bar{p}, \bar{q},\left(\bar{x}^{i}, \bar{z}^{i}\right)_{i \in \mathcal{I}}\right)$ be a fixed point of $\Psi_{M}$ such that,

$$
\bar{p} \gg 0, \quad \sum_{i \in \mathcal{I}} \bar{z}_{k}^{i} \leq 0, \quad \forall k \in \mathcal{J}_{a} ; \quad \sum_{i \in \mathcal{I}} \bar{x}_{s, l}^{i}<2 \bar{W}(M), \quad \forall(s, l) \in \mathcal{S} \times \mathcal{L} .
$$

Then, for any $j \in \mathcal{J}_{b}$ we have that,

$$
\bar{q}_{j}>0 \wedge \sum_{i \in \mathcal{I}} \bar{z}_{j}^{i}>0 \Longrightarrow \bar{q}_{j} \leq \bar{Q}
$$

Proof. Let $j \in \mathcal{J}_{b}$ such that $\bar{q}_{j}>0$ and $\sum_{i \in \mathcal{I}} \bar{z}_{j}^{i}>0$. Due to $\bar{p} \gg 0$, it follows from Assumption (A1)(iii) that $R_{j}(\bar{p}) \neq 0$. Hence, there is $s^{\prime} \in S$ such that $\sum_{r \in \mathcal{J}_{b}} R_{s^{\prime}, r}(\bar{p}) \geq R_{s^{\prime}, j}(\bar{p})>0$.

Fix $\delta \in(0,1)$. By Assumption (A4), it follows that

$\sum_{r \in \mathcal{J}_{b}} R_{s^{\prime}, r}(\bar{p})<(1+\delta)\left(\bar{p}_{s^{\prime}} \cdot Y_{s^{\prime}} \widehat{x}_{0}+\sum_{k \in \mathcal{J}_{a}} R_{s^{\prime}, k}(\bar{p}) \widehat{z}_{k}\right) \leq(1+\delta)\left(\bar{p}_{s^{\prime}} \cdot Y_{s^{\prime}} \widehat{x}_{0}+\left(\max _{k \in \mathcal{J}_{a}} \widehat{z}_{k}\right) \sum_{k \in \mathcal{J}_{a}} R_{s^{\prime}, k}(\bar{p})\right)$.

We affirm that,

$$
\bar{q}_{j} \leq(1+\delta)\left(\bar{p}_{0} \cdot \widehat{x}_{0}+\left(\max _{k \in \mathcal{J}_{a}} \widehat{z}_{k}\right) \sum_{k \in \mathcal{J}_{a}} \bar{q}_{k}\right) .
$$

Let $i$ be an agent that invests in asset $j$. Since long positions on $j$ can be reduced without affect the financial admissibility (Assumption (A4)(ii)), if the inequality above does not hold, then there is $\varepsilon>0$ such that, agent $i$ can reduce her long position on asset $j$ in $\varepsilon \bar{z}_{j}^{i}$ units, change her first-period consumption to $\bar{x}_{0}^{i}+(1+\delta) \varepsilon \bar{z}_{j}^{i} \widehat{x}_{0}$, and increase in $(1+\delta)\left(\max _{r \in \mathcal{J}_{a}} \widehat{z}_{r}\right) \varepsilon \bar{z}_{j}^{i}$ units the investment in each $k \in \mathcal{J}_{a}{ }^{14}$

With this strategy, $i$ changes her wealth at state of nature $s \in S$ by

$$
\left((1+\delta)\left(\bar{p}_{s} \cdot Y_{s} \widehat{x}_{0}+\left(\max _{k \in \mathcal{J}_{a}} \widehat{z}_{k}\right) \sum_{k \in \mathcal{J}_{a}} R_{s, k}(\bar{p})\right)-R_{s, j}(\bar{p})\right) \varepsilon \bar{z}_{j}^{i} \geq 0
$$

where the last inequality is a consequence of the super-replication property and holds as strict inequality for $s=s^{\prime}$. Since agent $i$ has locally non-satiated preferences (Assumption (A1)(i)), we contradict the optimality of $\left(\bar{x}^{i}, \bar{z}^{i}\right)$ on $C^{i}(\bar{p}, \bar{q}) \cap \mathbb{K}(M)$. We conclude that $\bar{q}_{j} \leq \bar{Q}$.

Lemma 4. Under Assumptions (A1)-(A4), for any $M>\bar{Q}$ the fixed points of $\Psi_{M}$ are competitive equilibria.

Proof. Given $M>\bar{Q}$, let $\left(\bar{p}, \bar{q},\left(\bar{x}^{i}, \bar{z}^{i}\right)_{i \in \mathcal{I}}\right)$ be a fixed point of $\Psi_{M}$. Adding first period budget constraints across agents, the definition of $\phi_{0, M}$ guarantees that,

$$
p_{0} \cdot \sum_{i \in \mathcal{I}}\left(\bar{x}_{0}^{i}-w_{0}^{i}\right)+q \cdot \sum_{i \in \mathcal{I}} \bar{z}^{i} \leq \bar{p}_{0} \cdot \sum_{i \in \mathcal{I}}\left(\bar{x}_{0}^{i}-w_{0}^{i}\right)+\bar{q} \cdot \sum_{i \in \mathcal{I}} \bar{z}^{i} \leq 0, \quad \forall\left(p_{0}, q\right) \in \mathcal{P}_{0} \times[0, M]^{\mathcal{J}_{b}} .
$$

Hence,

$$
\sum_{i \in \mathcal{I}}\left(\bar{x}_{0}^{i}-w_{0}^{i}\right) \leq 0, \quad \sum_{i \in \mathcal{I}} \bar{z}_{k}^{i} \leq 0, \forall k \in \mathcal{J}_{a},
$$

and $\bar{q}_{j}=M$ for every $j \in \mathcal{J}_{b}$ such that $\sum_{i \in \mathcal{I}} \bar{z}_{j}^{i}>0$. Furthermore, adding individual budget constraints at any state of nature in the second period, the definition of $\mathbb{K}(M)$ guarantees that,

$$
p_{s} \cdot \sum_{i \in \mathcal{I}}\left(\bar{x}_{s}^{i}-W_{s}^{i}\right) \leq \bar{p}_{s} \cdot \sum_{i \in \mathcal{I}}\left(\bar{x}_{s}^{i}-W_{s}^{i}\right) \leq \bar{W}(M), \quad \forall p_{s} \in \mathcal{P}_{1}, \forall s \in S
$$

\footnotetext{
${ }^{14}$ As the new strategy needs to be on $\mathbb{K}(M)$, the value of $\varepsilon$ may depend on $\left(M, \bar{q}_{j}, \bar{x}_{0}^{i},\left(\bar{z}_{k}^{i}\right)_{k \in \mathcal{J} a}, \bar{z}_{j}^{i}\right)$.
} 
We obtain that $\sum_{i \in \mathcal{I}} \bar{x}_{s, l}^{i}<2 \bar{W}(M), \forall(s, l) \in \mathcal{S} \times \mathcal{L}$, which implies that $\bar{p} \gg 0$. In another case, if $\bar{p}_{s, l}=0$ for some $(s, l) \in \mathcal{S} \times \mathcal{L}$, then Assumption (A1)(ii) guarantees that at least one agent can improve her utility by increasing her consumption without additional costs. A contradiction to the optimality of plans $\left(\bar{x}^{i}, \bar{z}^{i}\right)_{i \in \mathcal{I} \text {. }}$

The strict positivity of commodity prices has several consequences. First, by Assumption (A1)(iii) asset promises are non-trivial. Thus, (A1)(i) and the absence of restrictions on investment ensure that asset prices are strictly positive. Second, as $\left(\bar{p}, \bar{q},\left(\bar{x}^{i}, \bar{z}^{i}\right)_{i \in \mathcal{I}}\right)$ satisfies the hypotheses of Lemma 3 and $M>\bar{Q}$, we obtain that $\sum_{i \in \mathcal{I}} \bar{z}^{i} \leq 0$. Third, Assumption (A1)(i) guarantees that budget constraints are satisfied by equality.

We conclude that,

$$
(\bar{p}, \bar{q}) \in \mathbb{P}(\bar{Q}), \quad(\bar{p}, \bar{q}) \gg 0, \quad \sum_{i \in \mathcal{I}}\left(\bar{x}^{i}-\left(W_{s}^{i}\right)_{s \in \mathcal{S}}\right)=0, \quad \sum_{i \in \mathcal{I}} \bar{z}^{i}=0,
$$

and Assumption (A3) implies that $\left(\bar{x}^{i}, \bar{z}^{i}\right)_{i \in \mathcal{I}} \in \Omega(\bar{p}, \bar{q}) \cap \operatorname{int}(\mathbb{K}(M)){ }^{15}$

Since for any $i \in \mathcal{I}$ the allocation $\left(\bar{x}^{i}, \bar{z}^{i}\right)$ belongs to $C^{i}(\bar{p}, \bar{q}) \cap \operatorname{int}(\mathbb{K}(M))$, given $\left(x^{i}, z^{i}\right) \in C^{i}(\bar{p}, \bar{q})$ with $x^{i} \neq \bar{x}^{i}$ there exists $\lambda \in(0,1)$ such that $\lambda\left(\bar{x}^{i}, \bar{z}^{i}\right)+(1-\lambda)\left(x^{i}, z^{i}\right) \in C^{i}(\bar{p}, \bar{q}) \cap \mathbb{K}(M)$. The strict quasi-concavity of utility functions (Assumption (A1)(i)) implies that $V^{i}\left(\lambda\left(\bar{x}^{i}, \bar{z}^{i}\right)+(1-\lambda)\left(x^{i}, z^{i}\right)\right)>\min \left\{V^{i}\left(\bar{x}^{i}\right), V^{i}\left(x^{i}\right)\right\}$. Since $\left(\bar{x}^{i}, \bar{z}^{i}\right) \in \phi_{M}^{i}(\bar{p}, \bar{q})$, we obtain that $V^{i}\left(x^{i}\right)<V^{i}\left(\bar{x}^{i}\right)$.

Therefore, $\left(\bar{x}^{i}, \bar{z}^{i}\right)$ is an optimal choice for agent $i$ in $C^{i}(\bar{p}, \bar{q})$.

CASE II. Assume that (A1)(ii)(b) holds.

Denote by $\mathcal{E}$ the original economy and let $i$ be an agent with strictly monotonic preferences and interior endowments. For any $n \in \mathbb{N}$, consider the economy $\mathcal{E}_{n}$ obtained from $\mathcal{E}$ by just perturbing aggregated individual endowments, which become $W_{n}^{i}:=W^{i}$ and $W_{n}^{k}:=W^{k}+n^{-1} W^{i}, \forall k \neq i$.

It follows from Case I that, for every $n \in \mathbb{N}$, there exists an equilibrium for the economy $\mathcal{E}_{n}$ satisfying $\left(p(n), q(n),\left(x^{k}(n), z^{k}(n)\right)_{k \in \mathcal{I}}\right) \in \mathbb{P}(\bar{Q}) \times \mathbb{K}(\bar{Q})^{\mathcal{I}}$ and $(p(n), q(n)) \gg 0$. Also, since $\mathbb{P}(\bar{Q}) \times \mathbb{K}(\bar{Q})^{\mathcal{I}}$ is a compact set, there exists a subsequence $\left\{\left(p\left(n_{r}\right), q\left(n_{r}\right),\left(x^{k}\left(n_{r}\right), z^{k}\left(n_{r}\right)\right)_{k \in \mathcal{I}}\right)\right\}_{r \in \mathbb{N}} \subseteq\left\{\left(p(n), q(n),\left(x^{k}(n), z^{k}(n)\right)_{k \in \mathcal{I}}\right)\right\}_{n \in \mathbb{N}}$ converging to some $\left(\bar{p}, \bar{q},\left(\bar{x}^{k}, \bar{z}^{k}\right)_{k \in \mathcal{I}}\right) \in \mathbb{P}(\bar{Q}) \times \mathbb{K}(\bar{Q})^{\mathcal{I}}$. Notice that, by construction, $\left(\bar{x}^{k}, \bar{z}^{k}\right)_{k \in \mathcal{I}}$ satisfies market feasibility conditions and Assumption (A2) ensures that $\left(\bar{x}^{k}, \bar{z}^{k}\right) \in C^{k}(\bar{p}, \bar{q})$ for each agent $k \in \mathcal{I}$.

We affirm that $\left(\bar{x}^{i}, \bar{z}^{i}\right)$ is an optimal choice for agent $i$ at prices $(\bar{p}, \bar{q})$. Indeed, assume by contradiction that there is $\left(x^{i}, z^{i}\right) \in C^{i}(\bar{p}, \bar{q})$ such that $V^{i}\left(x^{i}\right)>V^{i}\left(\bar{x}^{i}\right)$. Then it follows from the continuity of preferences, the interiority of endowments $W^{i}$, and the convexity of $\Phi^{i}$ that there are $\theta \in(0,1)$ and $\rho \geq 0$ satisfying

$$
\begin{gathered}
V^{i}\left(\theta x^{i}+(1-\theta)\left(\frac{w_{0}^{i}}{2}, 0\right)\right)>V^{i}\left(\bar{x}^{i}\right), \\
\theta\left(x^{i}, z^{i}\right)+(1-\theta)\left(\left(\frac{w_{0}^{i}}{2}, 0\right),(-\rho)_{j \in \mathcal{J}_{a}}\right) \in \Phi^{i}(\bar{p}, \bar{q}), \\
0.25 \min _{(s, l) \in S \times \mathcal{L}} W_{s, l}^{i} \geq \rho \max _{p \in \mathcal{P}_{1}} \max _{j \in \mathcal{J}_{a}} R_{s, j}(p),
\end{gathered}
$$

\footnotetext{
${ }^{15}$ Along the proof of the Theorem, (A3) is only required to ensure that $\left[(\bar{p}, \bar{q}) \gg 0 \wedge\left(\bar{x}^{i}, \bar{z}^{i}\right){ }_{i \in \mathcal{I}} \in \Omega(\bar{p}, \bar{q})\right] \Longrightarrow$ $\left(\bar{x}^{i}, \bar{z}^{i}\right)_{i \in \mathcal{I}} \in \operatorname{int}(\mathbb{K}(M))$. In fact, if we change the upper bound $\bar{\Omega}(M)$ for an arbitrary positive number in the definition of $\mathbb{K}(M)$, then all the other arguments in the proof of Theorem 1 still hold.
} 
and, therefore,

$$
\left(x^{i}(\theta, \rho), z^{i}(\theta, \rho)\right):=\theta\left(x^{i}, z^{i}\right)+(1-\theta)\left(\left(\frac{w_{0}^{i}}{2}, 0\right),(-\rho)_{j \in \mathcal{J}_{a}}\right) \in \stackrel{\circ}{C}^{i}(\bar{p}, \bar{q}) .{ }^{16}
$$

Since $\left(p\left(n_{r}\right), q\left(n_{r}\right)\right) \rightarrow_{r}(\bar{p}, \bar{q})$ and $V^{i}\left(x^{i}(\theta, \rho)\right)>V^{i}\left(\bar{x}^{i}\right)$, the lower hemicontinuity of $\dot{C}^{i}$ (see the proof of Lemma 1) implies that for $r$ large enough there is $\left(\tilde{x}^{i}(r), \tilde{z}^{i}(r)\right) \in \stackrel{\circ}{C}^{i}\left(p\left(n_{r}\right), q\left(n_{r}\right)\right)$ such that $V^{i}\left(\tilde{x}^{i}(r)\right)>$ $V^{i}\left(x^{i}\left(n_{r}\right)\right)$, which contradicts the optimality of $\left(x^{i}\left(n_{r}\right), z^{i}\left(n_{r}\right)\right)$. Therefore, $\left(\bar{x}^{i}, \bar{z}^{i}\right)$ is an optimal choice for agent $i$ in $C^{i}(\bar{p}, \bar{q})$. Since $V^{i}$ is strictly increasing, we also conclude that $\bar{p} \gg 0 .{ }^{17}$

The strict positivity of commodity prices guarantees that every agent can obtain resources from selling her endowments. Thus, by analogous arguments to those made above for the case of agent $i$ - taking $\rho=0$ - we can prove that $\left(\bar{x}^{k}, \bar{z}^{k}\right)$ is an optimal choice for agent $k \neq i$ at prices $(\bar{p}, \bar{q})$.

We conclude that $\left(\bar{p}, \bar{q},\left(\bar{x}^{k}, \bar{z}^{k}\right)_{k \in \mathcal{I}}\right)$ is a competitive equilibrium for $\mathcal{E}$.

Proof of Corollary 1. To prove equilibrium existence, it is sufficient to guarantee that the superreplication property of Assumption (A4) is satisfied when commodity prices belongs to $\mathcal{P}$. Let $\mathcal{J}_{a}^{*} \subseteq \mathcal{J}_{a}$ be the set of unsegmented contracts that satisfies the requirements imposed in the statement of Corollary 1. Then, the super-replication property holds by choosing $\widehat{x}_{0}=0 ; \widehat{z}_{k}=0, \forall k \in \mathcal{J}_{a} \backslash \mathcal{J}_{a}^{*}$; and $\widehat{z}_{k}=\max _{s \in S_{k}} \max _{p \in \mathcal{P}}\left(\sum_{j \in \mathcal{J}_{b}} R_{s, j}(p) / R_{s, k}(p)\right), \forall k \in \mathcal{J}_{a}^{*}$.

Proof of Corollary 2. We will guarantee that the super-replication property holds when commodity prices belongs to $\mathcal{P}$. Since assets are real and payments are given in terms of commodities in $\mathcal{L}^{*}$, for any $j \in \mathcal{J} \backslash \mathcal{J}_{a}$ there is a function $A_{s, j}: \mathbb{R}_{+}^{\mathcal{L} \times \mathcal{S}} \rightarrow \mathbb{R}_{+}^{\mathcal{L}}$ such that $R_{s, j}(p)=p_{s} \cdot A_{s, j}(p)$ and $\left(A_{s, j}\right)_{l}=0, \forall l \notin \mathcal{L}^{*}$. Thus, when commodity prices belongs to $\mathcal{P}$, we can super-replicate financial payments of asset in $\mathcal{J} \backslash \mathcal{J}_{a}$ by choosing $\left(\widehat{z}_{k}\right)_{k \in \mathcal{J}_{a}}=0$ and $\widehat{x}_{0}=a(1, \ldots, 1)$, where $a>0$ satisfies

$$
\max _{l \in \mathcal{L}^{*}} \max _{(p, s) \in \mathcal{P} \times \widehat{S}} \sum_{j \in \mathcal{J} \backslash \mathcal{J}_{a}}\left(A_{s, j}(p)\right)_{l}<a \min _{(s, l) \in \widehat{S} \times \mathcal{L}^{*}} Y_{s}(l, l)
$$

and $\widehat{S}:=\bigcup_{j \in \mathcal{J}} S_{j}$ are the states of nature in which assets make promises.

Proof of Corollary 3. Since assets are backed by physical collateral, it is possible to super-replicate financial payments by choosing $\left(\widehat{z}_{k}\right)_{k \in \mathcal{J}_{a}}=0$ and $\widehat{x}_{0}=\sum_{j \in \mathcal{J}} C_{j}$. Therefore, Assumption (A4)(i) holds when commodity prices belongs to $\mathcal{P}$ and it suffices to obtain equilibrium existence as a consequence of the proof of the Theorem.

\footnotetext{
${ }^{16}$ At this stage, we do not know if $\bar{p}_{0} \neq 0$. Hence, to obtain an allocation in the interior of her choice set, agent $i$ may need to increase her debt on non-segmented assets by choosing $\rho>0$. This action is compatible with trading and budget constraints because $z^{i}$ was reduced in a $(1-\theta)$ percent and $W^{i} \gg 0$.

${ }^{17}$ Notice that, $\left(\bar{x}^{i}, \bar{z}^{i}\right) \in \operatorname{int}(\mathbb{K}(\bar{Q}))$ as a direct consequence of the market feasibility of $\left(\bar{x}^{k}, \bar{z}^{k}\right)_{k \in \mathcal{I}}$.
} 


\section{REFERENCES}

[1] Angeloni, L. and B. Cornet (2006): "Existence of financial equilibria in a multi-period stochastic economy," Advances in Mathematical Economics, 8, 933-955.

[2] Aouani, Z. and B. Cornet (2009): "Existence of financial equilibria with restricted participation," Journal of Mathematical Economics, 45, 772-786.

[3] Aouani, Z. and B. Cornet (2011): "Reduced equivalent form of a financial structure," Journal of Mathematical Economics, 47, 318-327.

[4] Balasko, Y., D. Cass, and P. Siconolfi (1990): "The structure of financial equilibrium with endogenous yields," Journal of Mathematical Economics, 19, 195-216.

[5] Border, K. (1985): "Fixed point theorems with applications to economics and game theory," Cambridge University Press, United Kigdom.

[6] Carosi, L., M. Gori, and A. Villanacci (2009): "Endogenous restricted participation in general financial equilibrium," Journal of Mathematical Economics, 36, 61-76.

[7] Cass, D. (1984): "Competitive equilibrium with incomplete financial markets", CARESS working paper.

[8] Cass, D. (2006): "Competitive equilibrium with incomplete financial markets", Journal of Mathematical Economics, 42, 384-405.

[9] Cass, D., P. Siconolfi, and A. Villanacci (2001): "Generic regularity of competitive equilibria with restricted participation," Journal of Mathematical Economics, 45, 787-806.

[10] Cea-Echenique, S., and J.P. Torres-Martínez (2014): "General equilibrium with endogenous trading constraints," SSRN working paper. Available at: http://dx.doi.org/10.2139/ssrn.2428354

[11] Cornet, B., and R. Gopalan (2010): "Arbitrage and equilibrium with portfolio constraints," Economic Theory, $45,227-252$.

[12] Geanakoplos, J., and W.R. Zame (2013): "Collateral equilibrium, I: a basic framework," Economic Theory, doi: $10.1007 /$ s00199-013-0797-4.

[13] Gori, M., M. Pireddu, and A. Villanacci (2013): "Existence of financial equilibria with endogenous short selling restrictions and real assets," Decisions in Economics and Finance, doi: 10.1007/s10203-013-0144-z.

[14] Gottardi, P., and T. Hens (1996): "The survival assumption and existence of competitive equilibria when asset markets are incomplete," Journal of Economic Theory, 71, 313-323.

[15] Hahn, G., and D.C. Won (2012): "Equilibrium theory with link portfolios," SSRN working paper. Available at: http://ssrn.com/abstract $=2140942$.

[16] Hahn, G., and D.C. Won (2007): "Constrained asset markets," SSRN working paper. Available at: http://ssrn.com/abstract $=1021631$.

[17] Hart, O. (1975): "On the optimality of equilibrium when the market structure is incomplete," Journal of Economic Theory, 11, 418-443.

[18] Hoelle, M., M. Pireddu, and A. Villanacci (2012): "Incomplete financial markets with real assets and wealthdependent credit limits," Krannert Working Paper Series, Paper No. 1271.

[19] Iraola, M.A., and J.P. Torres-Martínez (2014): "Equilibrium in collateralized asset markets: credit contractions and negative equity loans," Journal of Mathematical Economics, 55, 113-122.

[20] Martins-da-Rocha, F., L. Triki (2005): "Equilibria in exchange economies with financial constraints: beyond the Cass-trick," working paper, University Paris 1.

[21] Polemarchakis, H., and P. Siconolfi (1997): "Generic existence of competitive equilibria with restricted participation," Journal of Mathematical Economics, 28, 289-311. 
[22] Radner, R. (1972): "Existence of equilibrium of plans, prices, and price expectations," Econometrica, 40, 289303.

[23] Seghir, A., and J.P. Torres-Martínez (2011): "On equilibrium existence with endogenous restricted financial participation," Journal of Mathematical Economics, 47, 37-42.

[24] Siconolfi, P. (1989): "Equilibrium with asymmetric constraints on portfolio holdings and incomplete financial markets," in Non-Linear Dynamics in Economics and Social Sciences, M. Galeotti, L. Geronazzo, F. Gori editors, Societa' Pitagora, 271-292. 